This article was published in Journal of Chemical Education, 93(8), 14461451,2016

http://dx.doi.org/10.1021/acs.jchemed.5b00837

5 Preparation and characterization of polymeric nanoparticles: An Interdisciplinary Experiment

Maria J Ramalho ${ }^{1}$ and M Carmo Pereira ${ }^{1 *}$

Address: 1 LEPABE, Department of Chemical Engineering, Faculty of Engineering, University of Porto, Rua Dr. Roberto Frias, 4200-465 Porto, Portugal

ABSTRACT

In this work a laboratory experiment to introduce graduate students to nanotechnology is described. Students prepared poly(lactic-co-glycolic acid) (PLGA) nanoparticles using two different synthesis procedures, a single and a double emulsion-solvent evaporation method. The students also performed a physicochemical characterization of the prepared nanoparticles (NPs) by determining the hydrodynamic size and zeta potential values through Dynamic and Electrophoretic Light Scattering, respectively. The experiments were designed to be accomplished in two $180 \mathrm{~min}$ sessions and have been successfully conducted by 24 graduate students.

\title{
ABSTRACT GRAPHIC
}

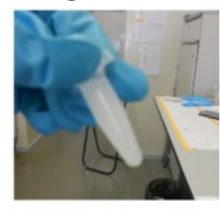

Single/double emulsion

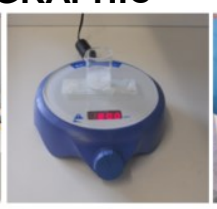

Organic solvent evaporation

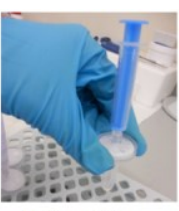

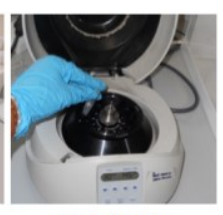

NPs collection

\section{KEYWORDS}

Graduate Education/Research; Nanotechnology; Colloids; Chemical Engineering; Interdisciplinary/Multidisciplinary; Laboratory Instruction; Hands-On 


\section{INTRODUCTION}

The term colloids or colloidal dispersions refers to heterogeneous systems composed of microscopic or submicroscopic particles of a dispersed material in a continuous phase. Many colloids are dispersions of nanoparticles (NPs). Nanoparticles are assemblies of atoms or molecules at nanometer scale with unique and useful technical properties, different from those of the bulk material, attributable to their small size. With the size reduction the surface/volume ratio is enlarged providing a larger reactive area of interface. The properties of this interface define the colloidal dispersions features.

These unique physicochemical characteristics justify the growing popularity of NPs in several applications as health, textile and food industries. ${ }^{1,2}$ Their use in the health sector has proven to be extremely advantageous in creating breakthroughs in diagnosis, treatment and monitoring of several diseases, enabling early diagnosis and the controlled delivery of drugs. ${ }^{3}$ Several materials have been widely studied and, in particular, poly(lactic-co-glycolic acid) (PLGA) proved to be an excellent candidate due to its well established clinical safety and advantageous physicochemical properties. ${ }^{4}$

The aim of this laboratory experiment is to provide graduate students with an introduction to the concepts of nanomaterials preparation using nanoparticle synthesis by the botton-up approach. By proposing an experiment of nanoencapsulation for upper-level undergraduate and graduate students in materials science, biotechnology, biomedical sciences, interfacial sciences, nanosciences; nanotechnology, pharmacy and supramolecular chemistry. This involves the preparation and characterization of PLGA NPs with the encapsulation of hydrophobic and hydrophilic molecules by the emulsionsolvent evaporation method. Pedagogical goals for this laboratory experiment include: the practice of new laboratory techniques for the encapsulation of different molecules in nanocarriers, and the contact with the classical laboratory techniques used for the characterization of nanocarriers. 
Despite the increasing use of nanomaterials in several industrial applications, not many laboratory experiments introducing different colloidal nanosytems in chemistry education curriculum are reported in the literature. A few experiments concerning metallic NPs such as gold, silver and zinc oxide NPs were already described in this journal..$^{5-8}$ Zinc oxide application in nanotechnology presents several advantages due to its characteristic properties. Hale and colleagues (2005) introduced a technique for the synthesis of zinc oxide NPs, in a third-year laboratory program for nanotechnology. The aim of this work was to provide a safer technique for the ones previously described. In this laboratory projects, students were presented with several theoretical concepts such as growth kinetics and nanoparticles physicochemical properties ${ }^{5}$. Guedens and co-workers (2014) introduced a microemulsion synthesis method for zinc hydroxide NPs to high school and undergraduate students. This experiment demonstrated that these NPs could be used in the sunscreens composition due to their ability to absorb UV radiation. ${ }^{6}$ Sharma and co-workers (2012) introduced students with an experimental procedure to prepare gold NPs using tea leaves. One of the major goals of this work was, beyond present concepts related to nanotechnology, to present the concept of green chemistry to chemistry undergraduate students. ${ }^{7}$ Orbaek and colleagues (2014) prepared a protocol for the production of silver NPs for an undergraduate chemical laboratory. The authors intended to provide students with 75 basic concepts of nanoparticles synthesis and physicochemical characterization. ${ }^{8}$ Also, only a few laboratory experiments to introduce polymer nanomaterials in the undergraduate and graduate chemistry curriculum are reported in the literature.9-12 One experiment involves the encapsulation of lipophilic compounds into polymeric micro and nanoparticles with the goal to introduce the emulsion-solvent evaporation method. ${ }^{9}$ In another work, a protocol for the design of a polymeric drug delivery system is described, focusing on the study of alginate polymerization reactions and its 
degradation and consequent release of the encapsulated compounds. ${ }^{10} \mathrm{~A}$ similar laboratory experiment was also described using gelatin. ${ }^{11}$ Other work describes the preparation methodology for PLGA films using a different synthesis technique from the one described in our work, but the main goal was to study the release kinetics of the prepared polymeric films. ${ }^{12}$ Unlike these studies, our work focuses not only on the nanoencapsulation of molecules with different chemical characteristics and the characterization techniques but also introduces the concepts of colloidal stability. Several concepts related to the physical-chemical characterization of nanoparticles were also presented. For these reasons, in this work a protocol for the preparation of PLGA NPs to be implemented for educational purposes is presented.

\section{EXPERIMENT OVERVIEW}

\section{Background}

PLGA NPs can be produced using several techniques. The characteristics of the obtained NPs, as size and structural organization, depend upon the chosen method and solvents. ${ }^{13-15}$ One of the most common methods, emulsion-solvent evaporation, is based on the shaping of the bulk material into nanostructures. Two types of the emulsionsolvent evaporation technique can be used: the single and double emulsion. The choice of each method depends upon the hydrophilicity of the drug to be encapsulated. ${ }^{15}$

In the single emulsion-solvent evaporation method, both drug (hydrophobic) and polymer are first dissolved in a water immiscible organic solvent, to form a single-phase solution. Then, this organic oil phase is emulsified in water containing an appropriate emulsifier. If the molecule to encapsulate is hydrophilic, it is necessary to prepare a double emulsion. This method is very similar to the single emulsion; however the main difference is the formation of two emulsions instead of a single one. Here, the drug is dissolved in the aqueous phase. To induce size reduction to the nanoscale, sonication is used. The size reduction is induced through a high-energy process, breaking up the 
primary droplets of dispersed oil into nanosized droplets. ${ }^{16,17}$ The subsequent removal of the organic solvent transforms droplets of dispersed phase into solid particles. ${ }^{18-20}$

In order to obtain a high efficiency, the organic solvent and the surfactant agent must be chosen carefully. A suitable solvent should be able to dissolve the polymer and to be poorly soluble in the continuous phase, and exhibit high volatility and low toxicity. ${ }^{19}$ As for the emulsifier, the most suitable surfactant is the one that promotes a greater reduction of the interfacial free energy. Thus avoiding the coalescence and agglomeration of drops, leading to a stabilized emulsion. ${ }^{19}$ Ethyl acetate and Pluronic F127 met the aforementioned requirements and were chosen for this experimental procedure.

\section{Reagents}

PLGA Resomer RG503H (50:50; MW 24,000 - 38,000), ethyl acetate, Pluronic F127 and coumarin-6 (MW 350.43) were purchased from Sigma-Aldrich (St. Louis, Mo., U.S.A). Trypan blue (MW 872.88) was acquired from Biochem Chemopharma (Loire, France). For TEM sample preparation, uranyl acetate (dehydrate, $424.146 \mathrm{~g} / \mathrm{mol}$ ) was purchased from Electron Microscopy Sciences (Hatfield, UK). All aqueous solutions were prepared in deionized and filtered ultrapure Milli-Q water (Milli-Q Academic, Millipore, France).

\section{Experimental details}

The experimental work was designed to be accomplished in two lab sessions. The synthesis protocol was conducted in a 180 min session and the characterization in another session. Twenty four students worked in groups of three. In the first lab session, all the student groups prepared NPs for each methodology protocol here described. In the second session, the student groups characterized the NPS in parallel by rotating the techniques. 
The single-emulsion solvent evaporation technique was used for the encapsulation of coumarin-6 (C6), a fluorescent probe used as a model molecule for hydrophobic drugs. This method is a suitable choice for encapsulating hydrophobic drugs. PLGA (10 $\mathrm{mg}$ ) was first dissolved in ethyl acetate $(0.1 \mathrm{~mL})$, and for encapsulation purpose $1 \mathrm{mg}$ of C6 was added. $0.2 \mathrm{~mL}$ of an aqueous phase of 1\% (w/v) Pluronic F127 was added dropwise to the prepared organic phase, and then the moisture was vortexed. As a result an oil-in-water emulsion was formed. In order to induce the nanosized droplets, a sonication process was carried out in an ultrasonic cleaner water bath $(45 \mathrm{kHz}, 5$ minutes). During the sonication, the eppendorf containing the emulsion should be immersed in a beaker with ice to avoid the water heating. The emulsion was then poured very fast into $2.5 \mathrm{~mL}$ of $0.1 \%$ (w/v) Pluronic F127 and stirred (800 rpm) at room temperature for 30 minutes with a nitrogen stream in order to completely evaporate the organic solvent. It is important to note that, this transfer step must be completed very quickly, in order to avoid PLGA NPs aggregation before being stabilized by the surfactant. The resulting suspension was filtered with Millex-GP Filter Units $(0.2 \mu \mathrm{m}$, polyethersulfone, Millipore Express, Ireland) and incubated at $4{ }^{\circ} \mathrm{C}$ overnight to avoid NPs aggregation and increase their stability.

\section{Synthesis of PLGA NPs - Encapsulation of hydrophilic molecules} encapsulating hydrophilic drugs. This method is very similar to the previous one, however the main difference is the formation of two emulsions instead of a single one. In this method, trypan blue (TB) - was used as a model for hydrophilic drugs. Firstly, $0.02 \mathrm{~mL}$ of an aqueous solution of $100 \mathrm{mg} / \mathrm{mL}$ TB was added dropwise to $0.1 \mathrm{~mL}$ of organic phase containing PLGA polymer dissolved in ethyl acetate $(100 \mathrm{mg} / \mathrm{mL})$. Subsequent vortex and sonication yielded the first emulsion, a water-in-oil emulsion. 
Then, $0.2 \mathrm{~mL}$ of the $1 \%(\mathrm{w} / \mathrm{v})$ Pluronic F127 aqueous solution was added dropwise to the prepared emulsion, and the remaining protocol was performed as already described. As a result, a water-in-oil-in-water emulsion is formed.

\section{Characterization of PLGA NPs}

The size, polydispersity index, zeta potential, and morphology were the parameters used to characterize the produced nanoparticles. The prepared NPs were collected by centrifugation (14000 rcf, $30 \mathrm{~min}$ ) and the obtained pellet was resuspended in ultrapure water. Both pellet and supernatant were saved for analysis.

Dynamic Light Scattering (DLS) is a well-known technique to determine the size of nanoparticles in dispersion or solution. In addition to the size, DLS also allows the determination of the polydispersity index (PdI), an indicative of the heterogeneity of NPs sizes in a suspension. ${ }^{21}$ For a near-monodisperse sample, a PdI of 0.1 or lower is expected. ${ }^{22}$ The presence of aggregates can distort the results, since the equipment analyzes them as a large size single particle, resulting in higher PdIs. ${ }^{23}$ In this work, DLS measurements were performed using a ZetaSizer Nano ZS (Malvern Instruments, Worcestershire, UK). All the determinations were performed in disposable cells (Sarstedt, Germany) and using water as dispersant medium. Mean values for each preparation were obtained by triplicate measurements. Each measurement was performed with 12 runs.

Electrophoretic light scattering (ELS) was used to evaluate the zeta potential, a key parameter for the NPs suspension stability. Particles with very positive/negative zeta potential values will repel each other and resist to aggregation. On the contrary, low absolute zeta potential values results in aggregation and flocculation. ${ }^{2,24}$ A physically stable nanosuspension will have a minimum zeta potential of absolute value of $30 \mathrm{mV}$ 13. Usually the laboratory equipment incorporates several techniques in a single 
compact unit. In fact, in this work ZetaSizer Nano ZS also provided measurements of zeta potential by ELS. It was used folded capillary cells from Malvern (Worcestershire, UK) and the dispersant medium was water. Mean values for each preparation were obtained by triplicate measurements. Each measurement was performed with 12 runs.

Transmission electron microscopy (TEM) is one of the most efficient and versatile tools for the characterization of nanoparticles morphology. The morphological examination of the NPs was performed by TEM Yeol Yem 1400 at an accelerating voltage of $80 \mathrm{kV}$ (Tokyo, Japan). For that purpose, the samples were prepared with negative staining to improve their visualization. $10 \mu \mathrm{L}$ of NPs suspension in ultrapure water were stained with $2 \%(\mathrm{v} / \mathrm{v})$ uranyl acetate for 45 seconds, immobilized on copper grids (Formvar/Carbon on 400 mesh Cu (50) from Agar Scientific), and air-dried for TEM visualization. ${ }^{25}$ Uranyl acetate is a heavy metal salt capable of scattering electrons, and therefore enhancing the image contrast. ${ }^{26}$

Fluorescence microscopy was used for the morphologic studies of the prepared NPs using an Eclipse Ti-S inverted microscope (Nikon, Carnaxide, Portugal). The fluorescent dyes entrapped in the NPs' matrix allow a fluorescent visualization of the PLGA NPs.

The effect of the suspension pH on the NPs surface charge was assessed by zeta potential measurements. After preparation, blank PLGA NPs were resuspended in aqueous buffers with different $\mathrm{pH}$ values $(\mathrm{pH}=4 ; 7$ and 10$)$ and their zeta potential values were determined.

\section{HAZARDS}

All reagents should be handled with students wearing gloves and lab coats. Ethyl acetate is toxic, highly flammable and slightly hazardous in case of skin or eye contact. Owing to the presence of its organic vapors, students should perform this experiment in 
a fume hood. Uranyl acetate is a highly toxic and radioactive material and therefore

215 should be handled with extreme care. Trypan blue is hazardous in case of ingestion. The other chemicals used do not have specific hazard recommendation.

\section{RESULTS AND DISCUSSION}

Students prepared and characterized PLGA nanoparticles obtained by a single or double emulsion-solvent evaporation method depending on the molecule's hydrophobicity. Figure 1 shows the picture of an obtained emulsion.

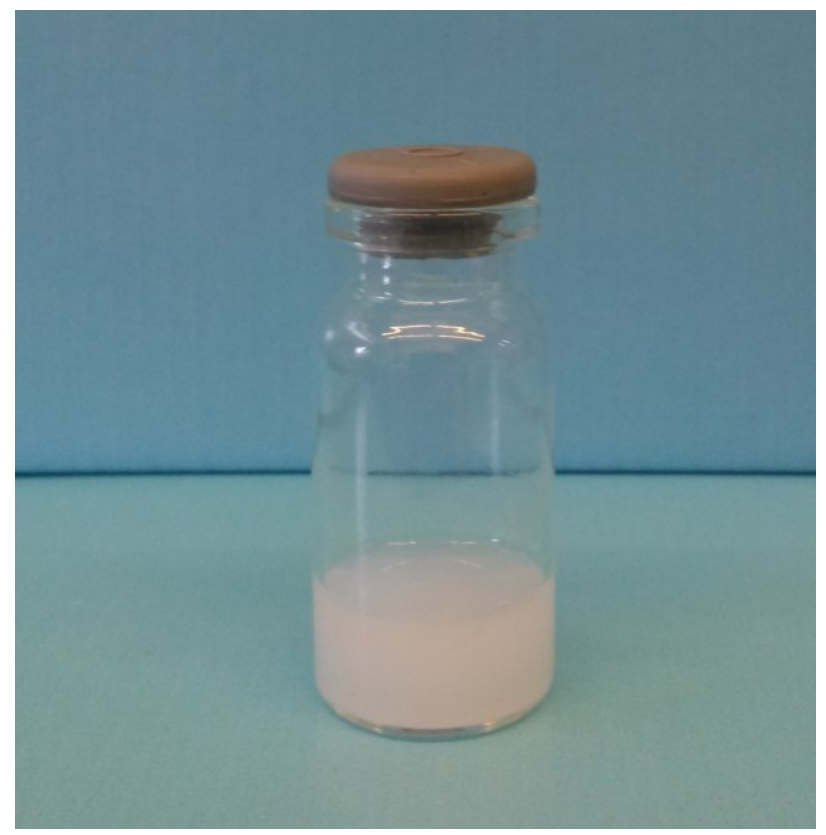

Figure 1. Colloidal suspension of PLGA NPs prepared by the single emulsion-solvent evaporation method.

The NPs obtained by both preparation methods exhibited mean diameters smaller than $200 \mathrm{~nm}$ as shown in figure 2 with a narrow size distribution (PdI $\leq 0.1)$. 


\section{Size distribution by intensity}

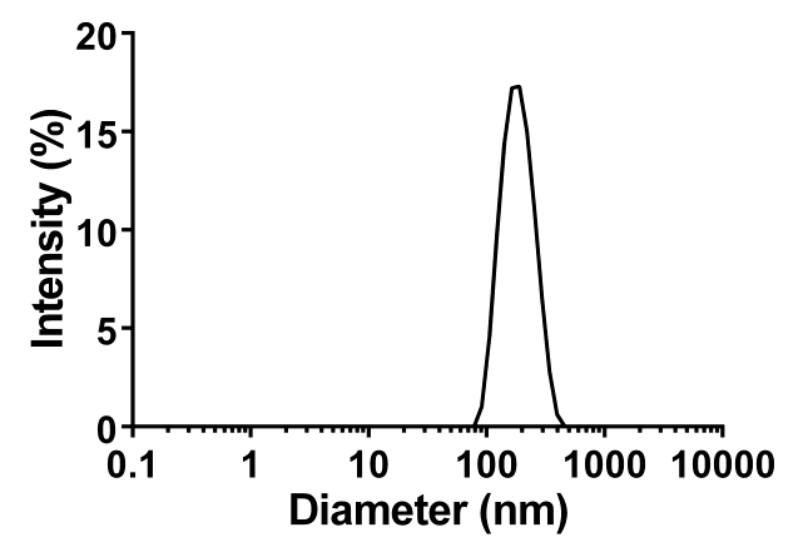

Figure 2. Hydrodynamic diameter of the PLGA NPs prepared by the single emulsion solvent evaporation method measured by DLS technique.

The emulsion technique yielded a well-stabilized nanosuspension, exhibiting high negative zeta potential values $(<-30 \mathrm{mV})$. This parameter plays a crucial role in maintaining the nanosuspension stabilization. Therefore, the colloidal stability results from the electrostatic stabilization provided by the surface charge due to the PLGA carboxylic end-groups, and the steric stabilization due to the Pluronic F127 layer adsorbed on the NPs surface. During particle formation, the stabilizer adsorbs onto the NP surface enhancing the colloidal stability acting as a mechanical barrier inhibiting the aggregation of the NPs. ${ }^{27}$ In figure 3, a morphologic examination performed by TEM shows this Pluronic layer surrounding PLGA NPs. Additionally, TEM analysis revealed spherical and uniform shaped nanoparticles. 


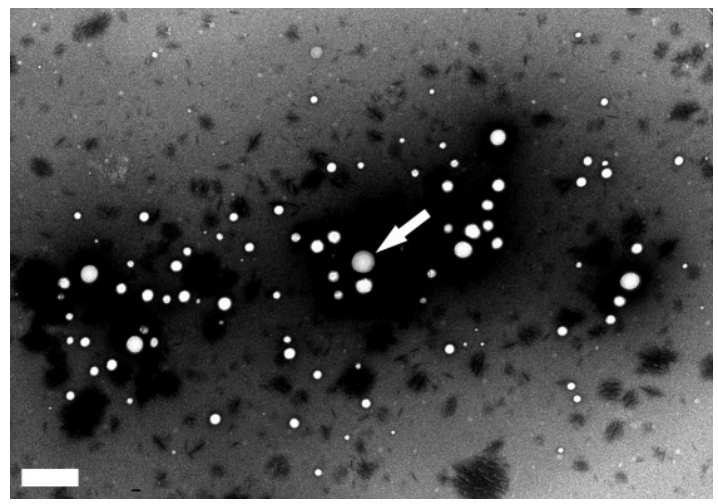

Figure 3.Transmission electron microscopy images of the PLGA nanoparticles prepared by the single emulsion solvent evaporation method - the scale bar corresponds to $500 \mathrm{~nm}$. The PLGA NPs appear as white spheres and the white arrow indicates the Pluronic layer surrounding the PLGA NP.

The zeta potential values of a colloidal nanosuspension depend on the NP surface charge and on the free salt ion concentration. As figure 4 shows, zeta potential of the PLGA NPs prepared using the single-emulsion method decreased with the decrease of $\mathrm{pH}$ value of the medium. The PLGA NPs acquired a positive charge at acidic $\mathrm{pH}$ due to the protonation of the carboxylic groups in PLGA. Oppositely, at neutral and basic $\mathrm{pH}$ most of surface charges of PLGA nanoparticles become negative. A similar behavior was observed in the PLGA NPs synthesized using the double-emulsion method.

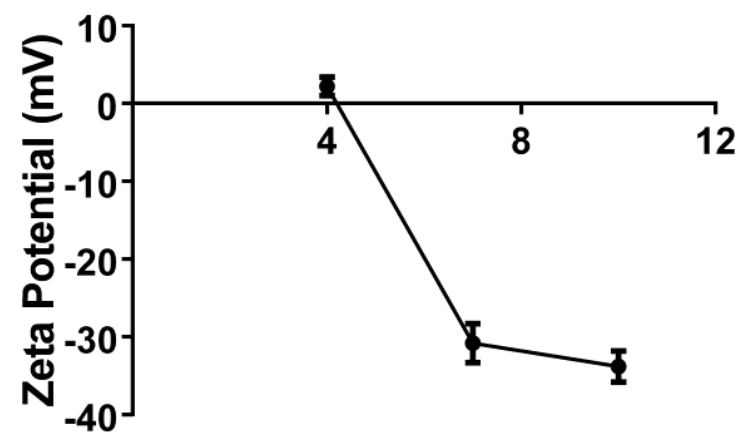

\section{$\mathrm{pH}$}

Figure 4. Variation of zeta potential of PLGA NPs with the $\mathrm{pH}$ values of the medium. Zeta potential was measured in 0.01 
At acidic $\mathrm{pH}$, a second population of NPs with sizes at the micron range appeared, suggesting that the NPs aggregation had occurred. Due to the protonation of the carboxylic groups in PLGA at acidic $\mathrm{pH}$ values, charge repulsion between the NPs /electrostatic stabilization is reduced.

Fluorescence microscopy analysis revealed spherical shaped nanoparticles as showed in figure 5. Although not having the necessary resolution for the NPs morphological characterization, this microscopy technique allowed the students to confirm the entrapment of the fluorescent marker into the NPs polymeric matrix.

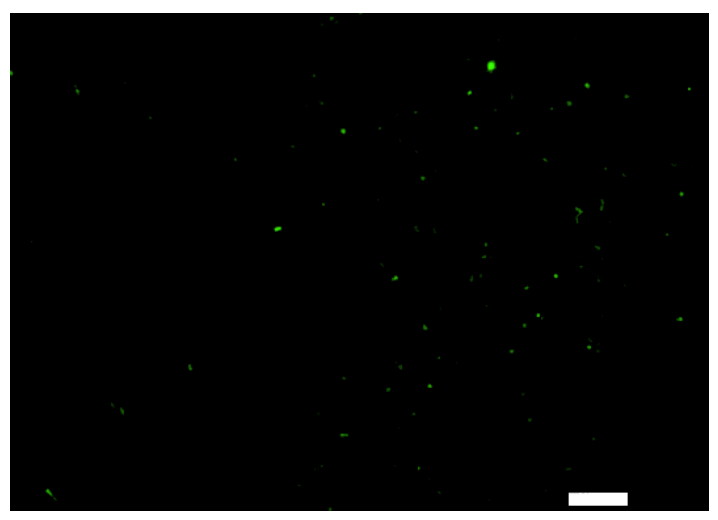

Figure 5. Fluorescence microscopy image of the prepared PLGA nanoparticles. The PLGA NPs were labeled with a fluorescent dye, coumarin-6. The scale bar corresponds to $10 \mu \mathrm{m}$.

As shown in the results presented above, both methods of synthesis, single and double-emulsion, yielded PLGA NPs with similar physicochemical characteristics. This allow us to conclude that both techniques are suitable methodologies for the preparation of PLGA NPs, and the choice of the most appropriated method to use depends only upon the hydrophilicity of the molecule to be encapsulated. 
This experiment was designed to be introduced to a nanotechnology course for graduate students. The theoretical concepts related with nanoparticles synthesis and characterization were presented to students in the corresponding lecture course and for that reason, students were expected to understand this laboratory work. However, some theoretical concepts were still provided to the students in the Background section in Notes for students (see Supporting Information file). Before the beginning of the experimental session, the instructor presented a brief introduction to the work and asked a few questions to the students about the experiment. At the end of the laboratory work, the students prepared a report with the obtained results and their discussion. The students were encouraged to compare their results to others described in the literature.

Summative assessment was achieved through the elaboration of the final report. The report showed that the students understood the theoretical concepts related to the preparation and characterization of nanoparticles. The percent of students answering correctly on report questions is presented in table 1.

Table 1. Comparison of Students' Correct Responses on Report Questions Probing NP Conceptual Understanding Questions and Statements for Student Response Correct Answers, \% $(N=24)$

1. What is the function of the Pluronic F127? 90

2. Are both of the prepared NPs colloidal suspensions stable? Justify.

80

3. Discuss about the surface charge of the PLGA nanoparticles.

90

4. Explain the changes in the surface charge of NPs with the variation of $\mathrm{pH}$ values.

85

Formative assessment was achieved by accompanying the students in the lab, carefully observing if students were able to successfully conduct all the tasks and understanding the theoretical fundaments of the characterization techniques. Comparing the results obtained by the students with the ones reported in the literature for PLGA NPs, shows that students successfully conducted the laboratory techniques 300 yielding nanoparticles with the expected physicochemical properties. The results from 
the formative assessment also showed that all the students were able to describe the conducted experiment, and successfully relate it to the analysis of the obtained results. The student's gain of a set of laboratory skills was also assessed. Table 2 lists these skills and the respective percentage of the students conducting the tasks successfully.

Table 2. Comparison of Laboratory Steps Students Completed Correctly

\begin{tabular}{lc} 
Laboratory Skills & Students Conducting the Step Correctly, $\%^{a}(N=24)$ \\
Calculations for solutions preparation & 80 \\
Balance use & 90 \\
Micropipette use & 80 \\
Emulsion preparation & 75 \\
Sonicator use & 100 \\
Filtration & 100 \\
\hline
\end{tabular}

${ }^{a}$ These results indicate the gain of the intended laboratory skills.

Although the authors priority in formative assessment was the students learned laboratory skills, student's perspective on the experiment was also assessed. Students did not exhibited unexpected difficulties during the experimental protocol execution, and seemed to be very interested in understanding the theoretical explanation for each protocol step. $90 \%$ of the students evaluate the experimental work as "stimulating", over $80 \%$ of the students indicated that the difficulty level of this activity was appropriated for their grade, and finally approximately $90 \%$ of students stated that this laboratory experiment increased their enthusiasm for nanotechnology.

\section{CONCLUSION}

A preparation method for PLGA NPs was described where students were able to have a first contact with nanomaterials and some important concepts related with other subjects like surface forces, emulsion techniques and characterization techniques. Preparation and characterization of PLGA NPs, for the encapsulation of both hydrophilic 
and hydrophobic molecules, has been the subject of many scientific articles ${ }^{18,20}$, but none of them have educational purpose targeting graduate students.

Although being a simple experimental procedure, this protocol involves many concepts of colloidal science and chemistry, allowing the students to explore the concept of hydrophobic/hydrophilic molecules, as to explore the physical and chemical conditions that control the stability and the aggregation of the NPs suspension.

Synthesis and characterization of nanocarriers and comparing results to literature were important research skills gained in this experimental work.

\section{ASSOCIATED CONTENT}

330 Supporting Information

Instructions for the students and notes for the instructor are available as

supplementary material.

\section{AUTHOR INFORMATION}

Corresponding Author

$335 \quad$ *E-mail: mcsp@fe.up.pt

\section{ACKNOWLEDGMENTS}

This work was financially supported by: Project POCI-01-0145-FEDER-006939 (Laboratory for Process Engineering, Environment, Biotechnology and Energy - LEPABE funded by FEDER funds through COMPETE2020 - Programa Operacional 340 Competitividade e Internacionalizaca, o (POCI) - and by national funds through FCT Fundação para a Ciência e a Tecnologia; FCT doctoral grant PD/BD/105984/2014. The authors acknowledge the graduate students of Master degree of Chemical Engineering 
(Nano Materials/Systems/Manufacturing, 5th year, 1st semester) and in the Junior University where this experiment was introduced.

\section{REFERENCES}

1. Barnes, G. ; Gentle, I. Chapter 1 - Introduction. in Interfacial Science: an introduction; Oxford University Press Inc.: New York, USA, 2011; pp 1-9.

2. Barnes, G. ; Gentle, I. Chapter 9 - The liquid-solid interface: adsorption; colloids. in Interfacial Science: an introduction; Oxford University Press Inc.: New York, USA, 2011; pp 242-287.

3. Kawasaki, E.S. ; Player, A. Nanotechnology, nanomedicine, and the development of new, effective therapies for cancer. Nanomedicine. 2005, 1(2), 101-9. doi:10.1016/j.nano.2005.03.002

4. Danhier, F.;Ansorena, E.;Silva, J.M.;Coco, R.;Le Breton, A.;Préat, V. PLGA-based nanoparticles: An overview of biomedical applications. Journal of Controlled Release. 2012, 161(2), 505-522. doi:10.1016/j.jconrel.2012.01.043

5. Hale, P.S.;Maddox, L.M.;Shapter, J.G.;Voelcker, N.H.;Ford, M.J.;Waclawik, E.R. Growth Kinetics and Modeling of ZnO Nanoparticles. Journal of Chemical Education. 2005, 82(5),

6. Guedens, W.J.;Reynders, M.;Van den Rul, H.;Elen, K.;Hardy, A.;Van Bael, M.K. ZnOBased Sunscreen: The Perfect Example To Introduce Nanoparticles in an Undergraduate or High School Chemistry Lab. Journal of Chemical Education. 2014, 91(2), 259-263. doi:10.1021/ed300851a

7. Sharma, R.K.;Gulati, S.;Mehta, S. Preparation of Gold Nanoparticles Using Tea: A Green Chemistry Experiment. Journal of Chemical Education. 2012, 89(10), 1316-1318. doi:10.1021/ed2002175

8. Orbaek, A.W.;McHale, M.M.;Barron, A.R. Synthesis and Characterization of Silver Nanoparticles for an Undergraduate Laboratory. Journal of Chemical Education. 2015,

9. Teixeira, Z.;Durán, N.;Guterres, S.S. Annatto Polymeric Microparticles: Natural Product Encapsulation by the Emulsion-Solvent Evaporation Method. Journal of Chemical Education. 2008, 85(7), 946. doi:10.1021/ed085p946

10. Friedli, A.C.;Schlager, I.R.;Stephen, W.W. Demonstrating Encapsulation and Release: A New Take on Alginate Complexation and the Nylon Rope Trick. Journal of Chemical Education. 2005, 82(7), 1017. doi:10.1021/ed082p1017

11. Sylman, J.L. ; Neeves, K.B. An Inquiry-Based Investigation of Controlled-Release Drug Delivery from Hydrogels: An Experiment for High School Chemistry and Biology. Journal of Chemical Education. 2013, 90(7), 918-921. doi:10.1021/ed300286v

12. Stevens, E.S.;Baumstein, K.;Leahy, J.-M.;Doetschman, D.C. Polymer-Plastics Experiments for the Chemistry Curriculum. Journal of Chemical Education. 2006, 83(10), 1531. doi:10.1021/ed083p1531

13. Hans, M.L.L., A.M. Biodegradable nanoparticles for drug delivery and targeting. Current Opinion in Solid State and Materials Science. 2002, 6, 319-327. doi:10.1016/S13590286(02)00117-1

14. Danhier, F.;Ansorena, E.;Silva, J.M.;Coco, R.;Le Breton, A.;Preat, V. PLGA-based nanoparticles: an overview of biomedical applications. J Control Release. 2012, 161(2), 505-22. doi:10.1016/j.jconrel.2012.01.043 
15. Makadia, H.K. ; Siegel, S.J. Poly Lactic-co-Glycolic Acid (PLGA) as Biodegradable Controlled Drug Delivery Carrier. Polymers (Basel). 2011, 3(3), 1377-1397. doi: $10.3390 /$ polym3031377

16. Kentish, S.;Wooster, T.J.;Ashokkumar, M.;Balachandran, S.;Mawson, R.;Simons, L. The use of ultrasonics for nanoemulsion preparation. Innovative Food Science \& Emerging Technologies. 2008, 9(2), 170-175. doi:10.1016/j.ifset.2007.07.005

17. Mahdi Jafari, S.;He, Y.;Bhandari, B. Nano-Emulsion Production by Sonication and Microfluidization-A Comparison. International Journal of Food Properties. 2006, 9(3), 475-485. doi:10.1080/10942910600596464

18. Frasco, M.F.;Almeida, G.M.;Santos-Silva, F.;Pereira, M.d.C.;Coelho, M.A.N. Transferrin surface-modified PLGA nanoparticles-mediated delivery of a proteasome inhibitor to human pancreatic cancer cells. Journal of Biomedical Materials Research Part A. 2015, 103(4), 1476-1484. doi:10.1002/jbm.a.35286

19. Li, M.;Rouaud, O.;Poncelet, D. Microencapsulation by solvent evaporation: state of the art for process engineering approaches. Int $J$ Pharm. 2008, 363(1-2), 26-39. doi:10.1016/j.ijpharm.2008.07.018

20. Ramalho, M.J.;Loureiro, J.A.;Gomes, B.;Frasco, M.F.;Coelho, M.A.N.;Pereira, M.C. PLGA nanoparticles as a platform for vitamin D-based cancer therapy. Beilstein Journal of Nanotechnology. 2015, 6, 1306-1318. doi:10.3762/bjnano.6.135

21. Tscharnuter, W. Photon Correlation Spectroscopy in Particle Sizing. in Encyclopedia of Analytical Chemistry, R.A. Meyers, Editor; John Wiley \& Sons, Ltd: Tarzana, California, USA, 2006; pp 5469-5485. doi:10.1002/9780470027318.a1512

22. Nobbmann, U.;Connah, M.;Fish, B.;Varley, P.;Gee, C.;Mulot, S.;Chen, J.;Zhou, L.;Lu, Y.;Shen, F.;Yi, J.;Harding, S.E. Dynamic light scattering as a relative tool for assessing the molecular integrity and stability of monoclonal antibodies. Biotechnol Genet Eng Rev. 2007, 24, 117-128. doi:10.1080/02648725.2007.10648095

23. Jiang, J.;Oberdörster, G.;Biswas, P. Characterization of size, surface charge, and agglomeration state of nanoparticle dispersions for toxicological studies. Journal of Nanoparticle Research. 2009, 11(1), 77-89. doi:10.1007/s11051-008-9446-4

24. Freire, J.;Domingues, M.;Matos, J.;Melo, M.;Veiga, A.;Santos, N.;Castanho, M.R.B. Using zeta-potential measurements to quantify peptide partition to lipid membranes. European Biophysics Journal. 2011, 40(4), 481-487. doi:10.1007/s00249-010-0661-4

25. Parveen, S. ; Sahoo, S.K. Long circulating chitosan/PEG blended PLGA nanoparticle for tumor drug delivery. European Journal of Pharmacology. 2011, 670(2-3), 372-383. doi:10.1016/j.ejphar.2011.09.023

26. Haschemeyer, R.H.M., R.J. Negative staining. in Principles and Techniques of Electron Microscopy - Biological Applications, M.A. Hayat, Editor; Van Nostrand Reinhold Company: NY, USA, 1972; pp 110-114.

27. Vandervoort, J. ; Ludwig, A. Biocompatible stabilizers in the preparation of PLGA nanoparticles: a factorial design study. International Journal of Pharmaceutics. 2002, 238(1-2), 77-92. doi:10.1016/S0378-5173(02)00058-3 